\title{
Increased FNDC5 (irisin) expression in subcutaneous adipose tissue in obese patients with type 2 diabetes one year after bariatric surgery.
}

Julia Muenzker ${ }^{1}$, Alexandra Harger ${ }^{1}$, Alexandru Tuca ${ }^{1}$, Lisa Lindheim¹ ${ }^{1}$ Verena Zachhuber ${ }^{1}$, Eva Svehlikova1 ${ }^{1}$, Oana Freisinger ${ }^{2}$, Johanna Fruhmann², Friedrich Tadler $^{3}$, Thomas R. Pieber ${ }^{1}$, Barbara Obermayer-Pietsch ${ }^{1}$

${ }_{1}^{1}$ Clinical Department for Endocrinology and Metabolism, Medical University of Graz, Austria

${ }^{2}$ Clinical Department of Surgery, Medical University of Graz, Austria

${ }^{3}$ Clinical Department of Surgery, Krankenhaus der Elisabethinen, Graz, Austria

\section{Background \& Aim}

Gastric bypass surgery improves glycaemic control, but the underlying mechanisms are incompletely understood. The aim of this study was to investigate potentially causal or associated alterations in gene expression of adipokines, myokines and hormones in the subcutaneous and visceral adipose tissue of diabetic and non-diabetic obese subjects before and after gastric bypass.

\section{Materials \& Methods}

Biopsy specimens from the adipose tissue of 12 diabetic and 12 non-diabetic patients at (subcutaneous, visceral) and one year after (subcutaneous) gastric bypass were analyzed with quantitative real-time PCR for the expression levels of various adipokines, myokines and hormones such as adiponectin, leptin, irisin (FNDC5), osteocalcin and osteopontin.

\section{Study Work Plan}

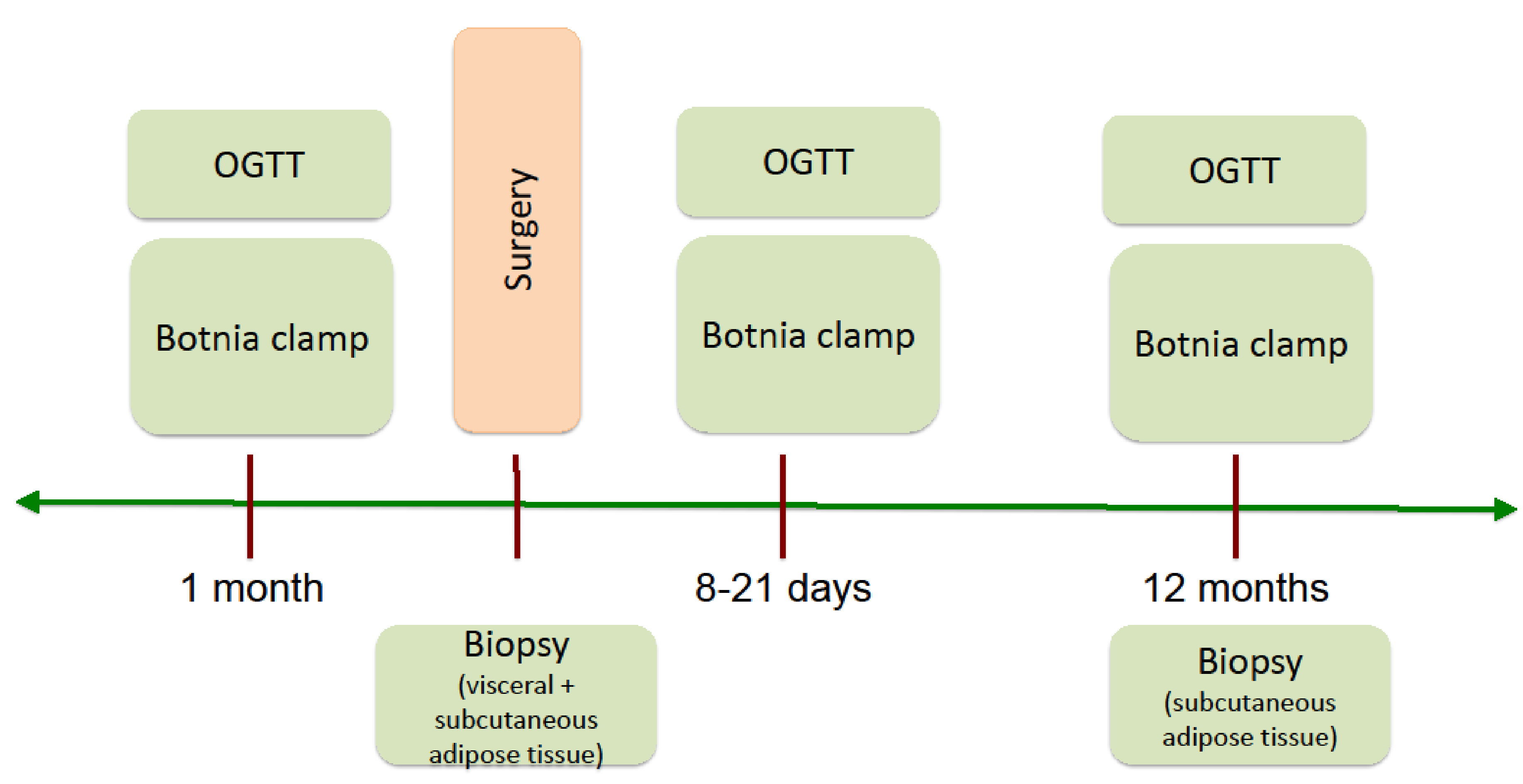

OGTT: oral glucose tolerance test; 180 min, 9 sampling points (assessment of glucose, insulin, C-peptide, proinsulin) Botnia clamp: IVGTT (intravenous glucose tolerance test; $60 \mathrm{~min}$ ) and subsequent hyperinsulinaemic- euglycaemic clamp (120 min)

\begin{tabular}{|c|c|c|c|c|c|c|}
\hline & \multicolumn{3}{|c|}{ Diabetics } & \multicolumn{3}{|c|}{ Non-Diabetics } \\
\hline & pre-OP & $\begin{array}{c}1 \text { year } \\
\text { postsurgery }\end{array}$ & $p$-value & pre-OP & $\begin{array}{c}1 \text { year } \\
\text { postsurgery }\end{array}$ & p-value \\
\hline Age (years) & $48 \pm 11$ & & & $46 \pm 8$ & & \\
\hline Weight (kg) & $129.63 \pm 18.52$ & $90.93 \pm 16.04$ & $<0.001$ & $125.17 \pm 18.08$ & $88.17 \pm 14.15$ & $<0.001$ \\
\hline BMI (kg/m2) & $46.06 \pm 5.57$ & $32.32 \pm 5.28$ & $<0.001$ & $44.07 \pm 5.26$ & $30.81 \pm 4.19$ & $<0.001$ \\
\hline HbA1c (\%) & $6.98 \pm 1.25$ & $5.98 \pm 0.78$ & 0.02 & $5.44 \pm 0.24$ & $5.24 \pm 0.25$ & 0.02 \\
\hline Fasting glucose $(\mathrm{mmol} / \mathrm{l})$ & $7.65 \pm 1.65$ & $5.57 \pm 1.36$ & $<0.001$ & $5.15 \pm 0.73$ & $5.40 \pm 1.02$ & $<0.001$ \\
\hline Fasting c-peptide $(\mathrm{ng} / \mathrm{ml})$ & $2.98 \pm 0.85$ & $1.48 \pm 0.43$ & $<0.001$ & $2.28 \pm 0.85$ & $1.07 \pm 0.29$ & $<0.001$ \\
\hline Fasting insulin $(\mathrm{mmol} / \mathrm{l})$ & $16.77 \pm 10.87$ & $4.67 \pm 2.85$ & $<0.001$ & $9.76 \pm 3.30$ & $3.03 \pm 2.24$ & $<0.001$ \\
\hline Fasting proinsulin (pmol/l) & $33.06 \pm 18.93$ & $6.59 \pm 2.56$ & $<0.001$ & $17.65 \pm 11.26$ & $4.10 \pm 1.60$ & 0.04 \\
\hline HOMA & $5.47 \pm 3.27$ & $1.41 \pm 1.10$ & 0.03 & $2.28 \pm 0.95$ & $0.65 \pm 0.57$ & 0.16 \\
\hline Insulin Sensitivity Index (ISI) & $-0.01 \pm 0.03$ & $0.07 \pm 0.03$ & $<0.001$ & $0.04 \pm 0.02$ & $0.10 \pm 0.01$ & $<0.001$ \\
\hline Early Insulin Response (EIR) & $0.17 \pm 0.17$ & $0.28 \pm 0.14$ & $<0.001$ & $0.56 \pm 0.24$ & $0.67 \pm 0.34$ & $<0.001$ \\
\hline Diabetes duration (years) & $8.3 \pm 6.32$ & & & & & \\
\hline
\end{tabular}

Clinical characteristics of patients at time of bariatric surgery and 1 year afterwards. Data are expressed as mean SD.

\section{Results}

Non-diabetic subjects were found to express significantly higher levels of FNDC5 in the subcutaneous adipose tissue than diabetic subjects before gastric bypass $(p=0.0167)$. FNDC5 expression in the subcutaneous adipose tissue of diabetic patients was significantly increased one year after surgery $(p<0.001)$. In non-diabetic subjects, there was no significant increase observable $(p=0.158)$. The increase in FNDC5 in diabetic subjects correlated with reduction in BMI (Pearson's $r=0.718$, $p=0.009$ ). No correlation was observed between FNDC5 expression and early insulin response or insulin sensitivity index.

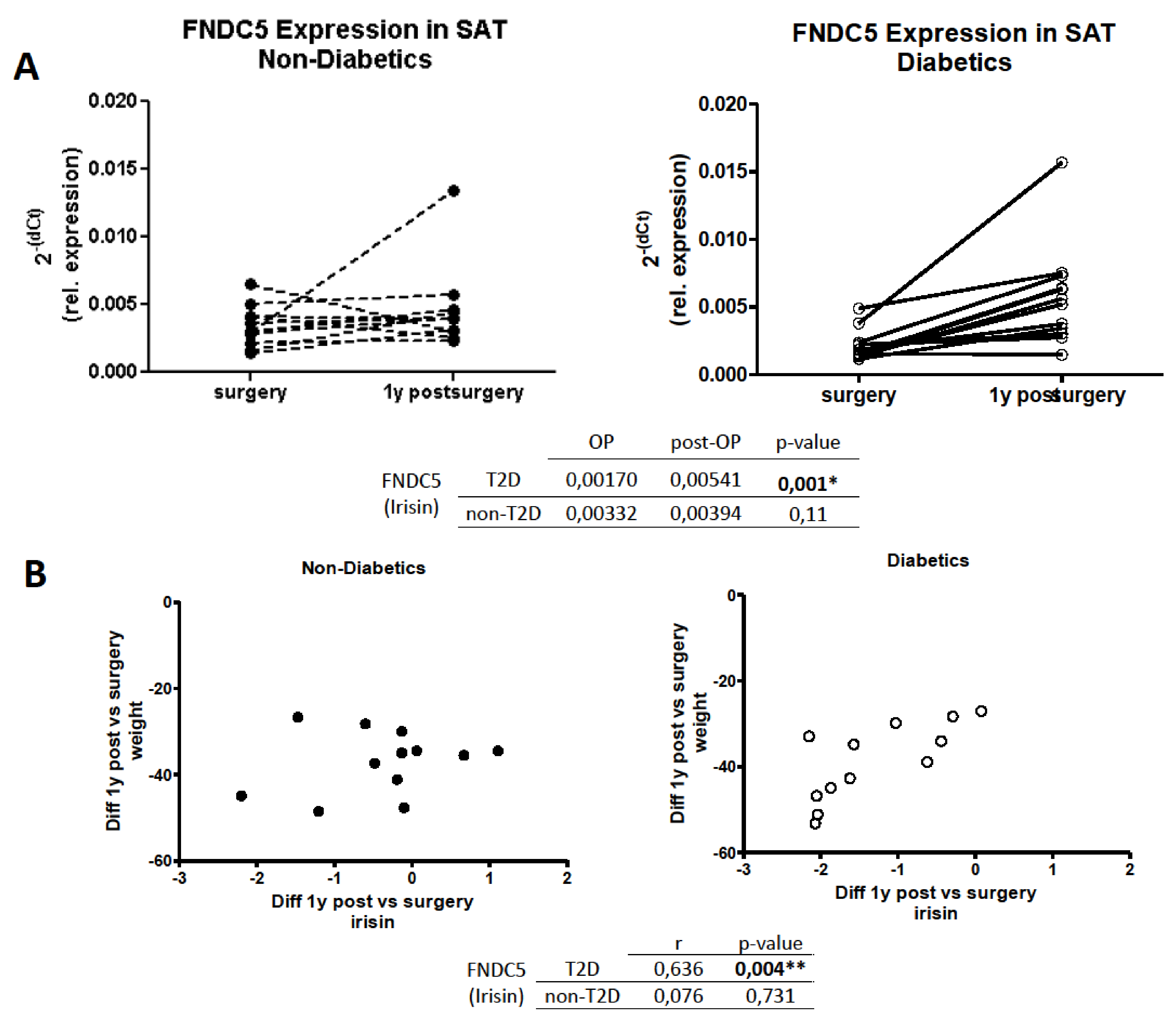

FNDC5 expresion in subcutaneous adipose tissue (SAT) at time of surgery and 1 year postsurgery (A), and correlation of weight reduction with difference in FNDC5 expression levels before and after surgery (B).

\section{Conclusion}

To our knowledge, this is the first study to investigate the role of the novel myokine irisin in bariatric surgery mediated weight loss. The results of this study demonstrate that exercise-independent weight loss increases (FNDC5) irisin expression levels in subcutaneous adipose tissue of diabetic subjects.

\section{Acknowledgements}

Contact:

julia.muenzker@medunigraz.at

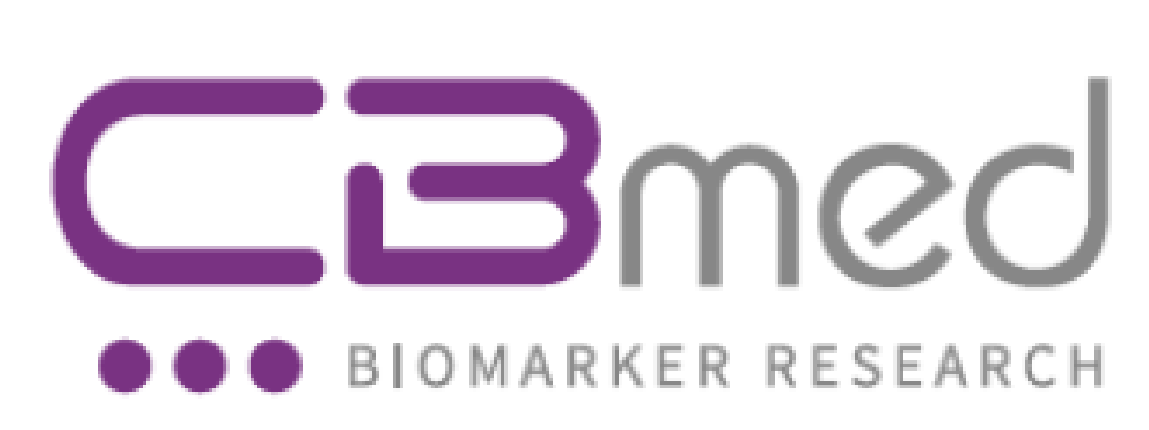

EFSD EFSD/MSD Clinical Research Grant 2009 Clinical Trial Registration: NCT 01271062 